\title{
Composición y estructura del ensamblaje de hormigas en diferentes usos del suelo en Santa Fe de Antioquia, Colombia
}

\section{Ant assemblage composition and structure in different land uses in Santa Fe de Antioquia, Colombia}

\author{
Mónica Estrada Agudelo ${ }^{1}$; Caty Milena Martínez-Bravo ${ }^{2}$; Néstor Javier Mancera-Rodríguez ${ }^{3 *}$
}

\begin{abstract}
${ }^{1}$ Ing. Agrónoma. Universidad Nacional de Colombia, Sede Medellín. Medellín - Antioquia, Colombia; e-mail: mestrada@invesa.com; Dhttps://orcid. org/0000-0003-0917-7577

²Bióloga, M.Sc. Universidad de Córdoba, Facultad de Ciencias Básicas, Grupo Biodiversidad Unicórdoba. Montería - Córdoba, Colombia; e-mail: catymilenam@ gmail.com; Dhttps://orcid.org/0000-0002-4349-4948

${ }^{3}$ Biólogo, Ph.D. Universidad Nacional de Colombia, Sede Medellín, Departamento de Ciencias Forestales. Medellín - Antioquia, Colombia; e-mail: njmancer@ unal.edu.co; Dhttps://orcid.org/0000-0002-7325-9588
\end{abstract}

*autor de correspondencia: njmancer@unal.edu.co

Cómo citar: Estrada Agudelo, M.; Martínez-Bravo, C.M.; Mancera-Rodríguez, N.J. 2019. Composición y estructura del ensamblaje de hormigas en diferentes usos del suelo en Santa Fe de Antioquia, Colombia. Rev. U.D.C.A Act. \& Div. Cient. 22(2):e1094. https://doi. org/10.31910/rudca.v22.n2.2019.1094

Artículo de acceso abierto publicado por Revista U.D.C.A Actualidad \& Divulgación Científica, bajo una licencia Creative Commons CC BY-NC 4.0

Recibido: Noviembre 6 de 2018

Aceptado: Agosto 24 de 2019

Editado por: Ingeborg Zenner de Polanía

\section{RESUMEN}

La Estación Agraria Cotové está ubicada en Santa Fe de Antioquia, zona de bosque seco tropical, en la que se han desarrollado actividades agropecuarias por más de 40 años, transformado sus ecosistemas originales y modificando las condiciones ambientales. Por lo anterior, este estudio tiene por objetivo evaluar la composición y la estructura de las hormigas en cinco sitios, con diferentes usos del suelo de la Estación Agraria: frutales, sistema silvopastoril, bosque secundario, pastos de corte y pastoreo con baja densidad de árboles. En cada uso del suelo, se ubicaron tres transectos de $100 \mathrm{~m}$ cada uno, al interior, interior-borde y borde, tomando una muestra cada $20 \mathrm{~m}$, a una profundidad de $10 \mathrm{~cm}$. Se identificaron las hormigas presentes, a nivel de morfoespecies y se clasificaron por gremios tróficos y por grupos funcionales. Se recolectaron 5.239 individuos, agrupados en 253 colonias, pertenecientes a 23 géneros y 37 morfoespecies de las subfamilias Myrmicinae, Ponerinae, Formicinae y Ecitoninae. La diversidad fue más alta en bosque secundario y frutales, mientras que la dominancia fue mayor en el sistema silvopastoril y en pastoreo con baja densidad de árboles. Entre bosque secundario y frutales, se encontró el mayor valor de morfoespecies compartidas. Los gremios tróficos mejor representados fueron omnívoros y depredadores y, por grupo funcional, se presentó una mayor proporción de especialistas de clima tropical y de myrmicinae generalistas.

Palabras clave: diversidad; gremios tróficos; sistemas agroforestales; conservación, bosque seco tropical.

\section{ABSTRACT}

The Agrarian Station Cotové is located in Santa Fe de Antioquia, an area of tropical dry forest in which agricultural activities have been carried out for more than 40 years, which have transformed their original ecosystems and modified the environmental conditions. 
Therefore, this study aims to evaluate the composition and structure of ants in five sites with different land uses: fruit trees, silvopasture, secondary forest, grass for cutting, and pastures with low density of trees. In each land use, three transects of $100 \mathrm{~m}$ each were located, inside, inside-edge, and edge, taking a sample every $20 \mathrm{~m}$ to a depth of $10 \mathrm{~cm}$. In each site, the ants were identified to morphospecies, and classified by trophic guilds and by functional groups. The diversity index for each land uses were determined. We collected 5239 ants, grouped in 253 colonies, belonging to 23 genera and 37 morphospecies of subfamilies Myrmicinae, Ponerinae, Formicinae y Ecitoninae. Species diversity was highest in secondary forest and fruit trees, while the dominance was higher in silvopasture and pastures with low density of trees. Secondary forest and fruit trees showed the highest value of shared morphospecies. The omnivorous and predator's trophic guilds were the best represented, and regarding functional groups, we found a high proportion of tropical climate specialists and Myrmicinae generalists.

Keywords: diversity; trophic guilds; agroforestry systems; conservation; tropical dry forest.

\section{INTRODUCCIÓN}

Las hormigas son un grupo ideal para realizar estudios en ecología de paisaje por ser importantes indicadores ecológicos, con amplia diversidad taxonómica, de gremios tróficos y funcionales, que operan en casi todos los estratos de los agroecosistemas (Arenas-Clavijo \& Armbrecht, 2018); asimismo, por su abundancia, la fidelidad ecológica de algunos grupos, la presencia de especies características de hábitats perturbados y de especies de importancia económica (Zabala et al. 2013).

En la región Andina de Colombia grandes áreas de bosque han sido convertidas a cultivos, como ampliación de la frontera agrícola y la conversión a pastos para la ganadería, lo que ha generado graves deterioros en la calidad de los suelos, pérdida de la biodiversidad y cambios en los sistemas ecológicos. Dentro de los ecosistemas más amenazados, se encuentran los bosques secos tropicales (bs-T) que, por la fertilidad de sus suelos, han sido punto de desarrollo de poblaciones humanas (Pennington et al. 2000). En este ecosistema, como en mucho otros, la expansión agrícola y pecuaria conduce a niveles importantes de degradación del suelo, lo que puede modificar la composición de las comunidades, reduciendo la abundancia y la riqueza de invertebrados y disminuyendo la calidad y cantidad de servicios ecológicos prestados por la diversidad biológica (Foley et al. 2011).

El departamento de Antioquia, se encuentra ubicado en la zona Andina de Colombia y a pesar que allí se concentra una alta diversidad biológica, las hormigas han sido poco estudiadas (Serna \& Vergara, 2007a). Se destacan los trabajos de Palacio (1997), en el Páramo de Frontino; de Toro \& Ortega (2006), en el Valle de Aburrá; de Serna \& Vergara (2001, 2007b), en el embalse hidroeléctrico Porce II; de Vergara et al. (2007), en el Arboretum de la Universidad Nacional de Colombia, sede Medellín y de Vanegas \& Sepúlveda (2008), en Santa Fe de Antioquia; este último realizó un inventario de hormigas en coberturas de frutales y bosque secundario.
La Estación Agraria Cotové está ubicada en una formación vegetal de bs-T, en el municipio de Santa Fe de Antioquia y se registra altamente afectada por la expansión ganadera, el incremento de fincas de recreo y áreas dedicadas a la construcción de proyectos urbanísticos (Martínez-Bravo et al. 2013). En la Estación, se han desarrollado actividades agropecuarias por más de 40 años, que han transformado sus ecosistemas originales, modificando las condiciones ambientales, aunque aún se conservan algunas áreas destinadas a la regeneración de cobertura arbórea. Por lo anterior, este estudio tuvo por objetivo evaluar la composición y la estructura, así como determinar los gremios tróficos y grupos funcionales del ensamblaje de hormigas en la Estación y su variación espacial, en cinco diferentes usos del suelo. Esta información ayudará a conocer la dinámica de las comunidades de hormigas de los sistemas productivos y establecer estrategias de manejo compatibles con la conservación de la diversidad biológica.

\section{MATERIALES Y MÉTODOS}

Localización y descripción del área de estudio. La Estación Agraria Cotové (EAC), se ubica en la vereda el Espinal del municipio de Santa Fe de Antioquia, Colombia (6 $6^{\circ} 33^{\prime} 32^{\prime \prime}$ N y $77^{\circ} 04^{\prime} 51^{\prime \prime}$ W; $530 \mathrm{~m}$ s.n.m.) y tiene una extensión de 121 hectáreas en una zona de vida de bs-T (Martínez-Bravo et al. 2013). El estudio de composición y de estructura del ensamblaje de hormigas, se realizó en cinco usos del suelo: frutales $(\mathrm{Fr})$, silvopastoreo $(\mathrm{Ss})$, bosque secundario (Bs), pastoreo con baja densidad de árboles $(\mathrm{Pb})$ y pastos de corte (Pc). La descripción detallada de los sistemas de uso de suelo, de las características físicas y químicas del suelo y de los valores de temperatura y precipitación promedio mensual, se pueden consultar en Jiménez-Heredia et al. (2010) y en Martínez-Bravo et al. (2013).

Métodos de campo y laboratorio. Se realizaron dos salidas de campo, la primera, octubre de 2007, período con lluvias prolongadas y suelos húmedos, y la segunda, en febrero de 2008, época con lluvias presentadas durante los días de muestreo y la irrigación de algunos de los sistemas evaluados, con la consecuencia de suelos muy húmedos. En cada uso del suelo, se establecieron tres transectos de $100 \mathrm{~m}$ cada uno, ubicados en el centro, a lo largo del borde y desde el borde hacia el interior del área de estudio. Cada transecto, se constituyó en una unidad de muestreo, dentro del cual, se tomaron 5 submuestras cada $20 \mathrm{~m}$, en las que se colectó la hojarasca y el primer horizonte del suelo (hasta una profundidad $10 \mathrm{~cm}$ ), en un área de $25 \mathrm{~cm}^{2}$. Estas submuestras, se depositaron en una bolsa plástica negra, debidamente rotulada, indicando: el tipo de uso del suelo, la ubicación del transecto (borde, interior, interior-borde), la distancia de la muestra $(0,20,40,60,80 \mathrm{~m})$ y la fecha. Se obtuvieron 3 muestras (1 por transecto), por uso del suelo por salida de campo, para un total de 6 muestras por uso de suelo y 30 muestras para los cinco usos.

Las muestras fueron procesadas en el laboratorio de Entomología, de la Facultad de Ciencias de la Universidad de Colombia, sede Medellín, donde se realizó la separación de los insectos, a través de embudo de Berlesse-Tullgren, por 24 horas; separación por estereomicroscopio y determinación taxonómica, utilizando las claves de Serna \& Vergara (2001) y Palacio \& Fernández (2003). 
Los grupos de macroinvertebrados colectados, se depositaron en la colección del museo de Entomología Francisco Luis Gallego, de la Universidad Nacional de Colombia, sede Medellín.

Análisis de datos: Se determinó el número de subfamilias, géneros y morfoespecies de hormigas y la presencia o ausencia de morfoespecies en cada muestra. De esta forma, cada registro corresponde a la aparición de una morfoespecie en una muestra determinada, sin importar la cantidad de ejemplares hallados en ésta. El número máximo de registros es de 6 por cada uso del suelo y 30 para toda la EAC. Con el número de registros, se calcularon los índices de Shannon-Wienner, Dominancia, Equidad de Pielou y el estimador de Chao2 para cada uso de suelo, utilizando el programa estadístico PAST (Hammer et al. 2001). Los valores de los índices de diversidad Shannon-Wienner y dominancia de Simpson entre usos de suelo, se compararon mediante pruebas de t de Hutcheson (Zar, 1999). La similitud entre morfoespecies presentes en los diferentes usos de suelo, se estimó mediante el índice de Jaccard. La rareza de morfoespecies encontradas en los distintos usos del suelo fue determinada como las especies que ocurrieron exclusivamente en un solo sitio de muestreo.

La estructura trófica de las hormigas, se determinó estableciendo para cada género la categoría del gremio trófico y el grupo funcional, para lo cual, se relacionaron datos biológicos, de microhábitat, observaciones de campo y la información presentada por Brown (2000) y Andersen (2000), quienes asocian el grupo funcional con el estrés y la perturbación ambiental. Los gremios tróficos fueron: omnívoros, depredadores y cultivadores de hongos y el grupo funcional en seis categorías: Camponotini subordinada (CS); Especialistas de Clima frío (ECF); Especialistas de Clima Tropical (ECT); Myrmicinae Generalista (MG); Oportunistas (O); Depredadores Especialistas (PE). Se calculó el número de registros y la proporción por gremio trófico y grupo funcional en cada uso de suelo en particular.

Para determinar diferencias en el número de registros entre subfamilias, gremios tróficos y grupos funcionales por uso de suelo, se compararon los valores de porcentaje de registros de cada uno de ellos entre las diferentes muestras tomadas, mediante análisis de varianza unidireccionales (ANOVA), validando que los datos cumplieran con los supuestos de normalidad (prueba de ShapiroWilk) y homocedasticidad (prueba de Levene) y comparaciones (post hoc), que fueron evaluadas, mediante la prueba de Tukey y cuando no cumplieron los supuestos, se utilizaron las comparaciones pareadas de Mann-Whitney, utilizando el programa estadístico PAST (Hammer et al. 2001).

\section{RESULTADOS Y DISCUSIÓN}

Composición de la comunidad de hormigas e Índices de diversidad: Durante el estudio, se capturaron 5.239 especímenes de hormigas, en 217 registros, para las 30 muestras estudiadas, agrupadas en 23 géneros y 37 morfoespecies de las subfamilias: Myrmicinae, Ponerinae, Formicinae y Ecitoninae (Tabla 1). La subfamilia Myrmicinae presentó un mayor número de géneros y de morfoespecies, $12(52,2 \%)$ y 23 (62,2\%), respectivamente, seguida en riqueza por la subfamilia Ponerinae, con 7 géneros $(30,4 \%)$ y 8 morfoespecies (21,6\%). La menor riqueza ocurrió en la subfamilia Ecitoninae, la cual, solo presentó un género $(4,3 \%)$ y una morfoespecie $(2,7 \%)$ (Tabla 1$)$, resultados coincidentes con Toro \& Ortega (2006), para áreas protegidas del Valle de Aburrá (Antioquia), donde hubo una distribución similar de riqueza, para estas cuatro subfamilias.

Para los cinco usos de suelo, se presentaron mayores registros de morfoespecies de la subfamilia Myrmicinae, siendo los registros similares entre usos de suelo (Figura 1). La subfamilia Ponerinae fue la segunda en porcentaje de registros para todos los usos, a excepción de Pc, donde fue Formicinae; en esta subfamilia, se registró diferencia entre los usos de suelo Pc y Bs (Mann-Whitney, $\mathrm{P}=0,012$ ) y entre $\mathrm{Pc}$ y $\mathrm{Pb}$ (Mann-Whitney, $\mathrm{P}=0,012)$. Se observó que para los usos de frutales (Fr) y silvopastoreo $(\mathrm{Ss})$ no se colectaron morfoespecies de la subfamilia Ecitoninae (Figura 1). Los resultados encontrados en los usos de suelo Fr y Bs concuerdan con Vanegas \& Sepúlveda (2008), quienes encontraron que en estos lugares los géneros de hormigas más comunes correspondieron a las subfamilias Myrmicinae y Ponerinae.

El uso de suelo con mayor riqueza fue bosque secundario, con 29 morfoespecies y 68 registros, seguido por frutales, con 23 morfoespecies y 54 registros, siendo el sistema silvopastoril el de menor riqueza, con 11 morfoespecies y 21 registros (Tabla 2). Este resultado es debido a que, en la medida que los hábitats presentan mayor producción primaria neta, frecuentemente, tienen mayor número de colonias de hormigas (Kaspari, 2003). Esta cobertura ofrece microclimas apropiados, menor variación de temperatura, que lleva a menor velocidad de desecación y niveles altos de humedad más estables, con oferta alimentaria abundante y estable, además de variedad de refugios para el establecimiento de las colonias de hormigas (Pardo et al. 2006), con relación a otros usos del suelo como los potreros para ganadería. Algunos trabajos que relacionan la distribución de especies de hormigas con gradientes sucesionales o usos del suelo han concluido que, con el aumento en la complejidad estructural del ecosistema, también aumenta la riqueza y la diversidad de especies (Bustos \& Chacón, 1997). Vanegas \& Sepúlveda (2008) registraron para el uso frutales $(\mathrm{Fr})$, un total de 16 morfoespecies y para Bosque secundario (Bs), 18 morfoespecies.

El número de morfoespecies observadas (37) para la Estación Agraria Cotové (EAC) es similar al estimador de riqueza Chao2, que arrojó un valor esperado de 37,7 especies. Se debe destacar que esta estimación corresponde a las especies de hábito estrictamente ligado al suelo. El índice de Shannon-Wienner presentó el mayor valor en Bs, seguido de Fr, usos de suelo entre los que no se encontró diferencias significativas. A su vez, estos usos presentaron diversidad mayor que los usos $\mathrm{Pc}$ y $\mathrm{Pb}$ con diferencias significativas $(\mathrm{p}<0,05)$ y el menor valor de diversidad, se presentó en el uso de suelo Ss (Tabla 2). La mayor diversidad observada en Bs y Fr con relación a los otros usos de suelo puede ser explicada por la incidencia negativa de las perturbaciones, como el pastoreo sobre los usos de $\mathrm{Pc}, \mathrm{Pb}$ y Ss, dado que la actividad pecuaria altera las propiedades 
Tabla 1. Número de registros de morfoespecies de hormigas presentes en los diferentes usos de suelo de la Estación Agraria Cotové (EAC).

\begin{tabular}{|c|c|c|c|c|c|c|c|c|c|}
\hline Subfamilia & Morfoespecie & Gremio trófico & $\begin{array}{c}\text { Grupo } \\
\text { Funcional }\end{array}$ & $\mathrm{Fr}$ & Ss & Bs & $\mathrm{Pb}$ & Pc & EAC \\
\hline Ecitoninae & Labidus msp1 & Depredadores & ECT & & & 2 & 1 & 1 & 4 \\
\hline \multirow{5}{*}{ Formicinae } & Acropyga msp1 & Omnívoros & CS & & & 4 & & 1 & 5 \\
\hline & Brachymyrmex msp1 & Omnívoros & ECT & & & & & 2 & 2 \\
\hline & Brachymyrmex msp2 & Omnívoros & ECT & & 2 & 1 & & & 3 \\
\hline & Brachymyrmex msp3 & Omnívoros & ECT & & & & & 2 & 2 \\
\hline & Paratrechina msp1 & Omnívoros & $\mathrm{O}$ & 1 & & 2 & 1 & 1 & 5 \\
\hline \multirow{23}{*}{ Myrmicinae } & Crematogaster msp1 & Omnívoros & MG & 1 & 1 & & 1 & 1 & 4 \\
\hline & Cyphomyrmex msp1 & $\begin{array}{l}\text { Cultivadores } \\
\text { de hongos }\end{array}$ & ECT & & & 2 & & & 2 \\
\hline & Myrmicocrypta msp1 & $\begin{array}{l}\text { Cultivadores } \\
\text { de hongos }\end{array}$ & ECT & & & 3 & & & 3 \\
\hline & Mycocepurus msp1 & $\begin{array}{l}\text { Cultivadores } \\
\text { de hongos }\end{array}$ & ECT & 1 & 1 & & & & 2 \\
\hline & Octostruma msp1 & Depredadores & $\mathrm{C}$ & 1 & 1 & 3 & & & 5 \\
\hline & Pheidole msp1 & Omnívoros & MG & 2 & & 1 & 1 & 3 & 7 \\
\hline & Pheidole msp2 & Omnívoros & MG & 3 & & & 3 & 3 & 9 \\
\hline & Pheidole msp3 & Omnívoros & MG & 1 & & 2 & 1 & 1 & 5 \\
\hline & Pheidole msp4 & Omnívoros & MG & 3 & 2 & 2 & 3 & 3 & 13 \\
\hline & Pheidole msp5 & Omnívoros & MG & 4 & 1 & 4 & & 2 & 11 \\
\hline & Pheidole msp6 & Omnívoros & MG & 2 & 2 & 1 & & & 5 \\
\hline & Pheidole msp7 & Omnívoros & MG & 2 & & & & & 2 \\
\hline & Pheidole msp8 & Omnívoros & MG & & & 1 & & & 1 \\
\hline & Pyramica msp1 & Depredadores & $\mathrm{PE}$ & & & 1 & & & 1 \\
\hline & Rogeria msp1 & Omnívoros & ECT & 3 & & 2 & & 1 & 6 \\
\hline & Solenopsis msp1 & Omnívoros & ECT & 6 & 5 & 6 & 5 & 6 & 28 \\
\hline & Solenopsis $\mathrm{msp} 2$ & Omnívoros & ECT & 2 & & 3 & 2 & 2 & 9 \\
\hline & Solenopsis $\mathrm{msp} 3$ & Omnívoros & ECT & 4 & 1 & 2 & 2 & 5 & 14 \\
\hline & Strumigenys msp1 & Depredadores & $\mathrm{C}$ & 3 & & 6 & 2 & 2 & 13 \\
\hline & Strumigenys msp2 & Depredadores & $\mathrm{C}$ & 4 & & 1 & 1 & & 6 \\
\hline & Strumigenys msp3 & Depredadores & $\mathrm{C}$ & 2 & & 1 & & & 3 \\
\hline & Tetramorium msp1 & Depredadores & $\mathrm{O}$ & & & & & 2 & 2 \\
\hline & Wasmannia msp1 & Omnívoros & ECT & 4 & 1 & 3 & & 2 & 10 \\
\hline \multirow{8}{*}{ Ponerinae } & Anochetus msp1 & Depredadores & $\mathrm{PE}$ & 1 & & 2 & & & 3 \\
\hline & Amblyopone msp1 & Depredadores & $\mathrm{C}$ & & & 1 & & & 1 \\
\hline & Discothyrea msp1 & Depredadores & $\mathrm{C}$ & & & 2 & & & 2 \\
\hline & Ectatomma msp1 & Depredadores & $\mathrm{O}$ & 1 & & & 1 & 2 & 4 \\
\hline & Gnamptogenys msp1 & Depredadores & ECT & & & 1 & & & 1 \\
\hline & Hypoponera msp1 & Depredadores & $\mathrm{C}$ & 2 & 4 & 6 & 6 & 1 & 19 \\
\hline & Pachycondyla msp1 & Depredadores & $\mathrm{PE}$ & & & 2 & & & 2 \\
\hline & Pachycondyla msp2 & Depredadores & $\mathrm{PE}$ & 1 & & 1 & 1 & & 3 \\
\hline \multicolumn{3}{|c|}{ TOTAL } & & 54 & 21 & 68 & 31 & 43 & 217 \\
\hline
\end{tabular}

Fr: Frutales; Ss: Sistema silvopastoril; Bs: Bosque secundario; Pb: Potreros de ganadería en baja densidad de árboles; Pc: Pastos de corte; EAC: Estación Agraria Cotové. CS: Camponotini subordinada; ECF: Especialistas de Clima frío; ECT: Especialistas de Clima Tropical; MG: Myrmicinae Generalista; O: Oportunistas; PE: Depredadores Especialistas. 


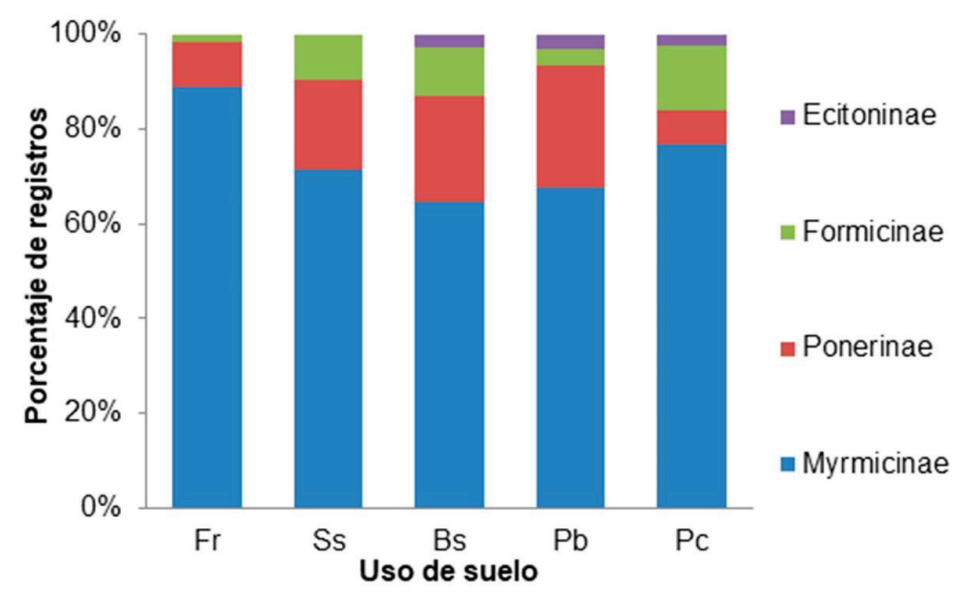

Figura 1. Porcentaje de morfoespecies por subfamilia en los cinco usos de suelo de la Estación Agraria Cotové. Fr: Frutales; Ss: Sistema silvopastoril; Bs: Bosque secundario; Pb: Potreros de ganadería en baja densidad de árboles; Pc: Pastos de corte.

Tabla 2. Índices de diversidad de hormigas presentes en los diferentes usos de suelo del Centro Agropecuario Cotové.

\begin{tabular}{|l|c|c|c|c|c|c|}
\hline & Fr & Ss & Bs & Pb & Pc & EAC \\
\hline Número de morfoespecies (S) & 23 & 11 & 29 & 15 & 20 & 37 \\
\hline Número de registros (Nr) & 54 & 21 & 68 & 31 & 43 & 217 \\
\hline Número de individuos (N) & 741 & 214 & 2048 & 1688 & 548 & 5239 \\
\hline Número de subfamilias & 3 & 3 & 4 & 4 & 4 & 4 \\
\hline Diversidad de Shannon (H') & $2,98 \mathrm{a}$ & $2,20 \mathrm{c}$ & $3,18 \mathrm{a}$ & $2,48 \mathrm{~b}$ & $2,83 \mathrm{~b}$ & 3,25 \\
\hline Dominancia (D) & 0,06 & 0,14 & 0,05 & 0,10 & 0,07 & 0,05 \\
\hline Índice de Simpson (1-D) & $0,94 \mathrm{a}$ & $0,86 \mathrm{~b}$ & $0,95 \mathrm{a}$ & $0,90 \mathrm{a}$ & $0,93 \mathrm{a}$ & 0,95 \\
\hline Equidad de Pielou & 0,95 & 0,92 & 0,95 & 0,92 & 0,95 & 0,90 \\
\hline Estimador de riqueza Chao2 & 27,5 & 15,7 & 36,2 & 18,6 & 21,6 & 37,7 \\
\hline
\end{tabular}

Fr: Frutales; Ss: Sistema silvopastoril; Bs: Bosque secundario; Pb: Potreros de ganadería en baja densidad de árboles; Pc: Pastos de corte; EAC: Estación Agraria Cotové. CS: Camponotini subordinada; ECF: Especialistas de Clima frío; ECT: Especialistas de Clima Tropical; MG: Myrmicinae Generalista; O: Oportunistas; PE: Depredadores Especialistas. Letras diferentes en la misma fila indican diferencias significativas $(\mathrm{p}<0,05)$.

del suelo, la estructura de la vegetación, los recursos alimenticios y las poblaciones de competidores y depredadores de hormigas, lo cual, puede tener efectos complementarios sobre las comunidades de hormigas. Los altos valores de diversidad obtenidos para bosque secundario y frutales coinciden con lo registrado por Vanegas \& Sepúlveda (2008), en estos mismos usos de suelo, en el que destacan que la zona de cultivo de mango, que corresponde al uso Fr, no está sometido a prácticas de manejo intensivas, tales como podas, desyerbas y fumigaciones constantes, lo que permite tener una diversidad mayor que otros usos dedicados a la ganadería.

La dominancia presentó valores altos en los usos de suelo Ss $(\mathrm{D}=0,14)$ y $\mathrm{Pb}(\mathrm{D}=0,10)$, por el predominio de los géneros Solenopsis e Hypoponera; esta dominancia, se puede dar porque se disminuyen los enemigos naturales, los cuales, se pueden encontrar en menor proporción o por aumento en los recursos de hábitat y alimenticios para estos géneros (Serna \& Vergara, 2007b). Para el índice de Simpson, se encontró un menor valor en el uso de suelo Ss, con diferencias significativas $(\mathrm{p}<0,05)$, respecto a los demás usos de suelo (Tabla 2). Finalmente, el índice de equidad de Pielou osciló entre 0,92, para los usos Ss y Pc y 0,95, para Bs, Fr y Pb (Tabla 2). Estos resultados coinciden con lo reportado por Serna \& Vergara (2007b), quienes plantean que este índice mostró mayor equidad en zonas de bosque y menor en áreas de pastizal, lo que indica que hay pérdida de diversidad, a medida que se incrementa el disturbio.

La composición taxonómica por morfoespecies entre los usos de suelo, de acuerdo con el índice de similitud de Jaccard, mostró una mayor similitud de morfoespecies entre $\mathrm{Pb}$ y $\mathrm{Pc}$, con un valor del $59,1 \%$, seguida de los usos Fr y Pb, con 58,3\% y de Fr y Pc, con 
53,6\%. Los altos niveles de similitud entre los sitios pueden estar influenciados por el relativo acercamiento entre los diferentes usos de suelo evaluados, como el caso de $\mathrm{Pb}$ y Pc y que, muchas especies de hormigas, en sus vuelos nupciales, pueden recorrer importantes distancias, así como también por ser hábitats sometidos al mismo tipo de perturbación, por actividades ganaderas. La menor similitud entre usos de suelo, se presentó entre $\mathrm{Ss}$ y $\mathrm{Pb}$ y entre $\mathrm{Ss}$ y $\mathrm{Pc}$, con un valor del $23,8 \%$ y $29,2 \%$, respectivamente. La poca similitud entre estos dos lugares, a pesar de ser dos sitios con similar manejo y capacidad de carga de ganado (2UGG/ha), se debe a que existen diferencias en cuanto a la densidad de árboles y cobertura vegetal en general, lo que puede incidir en la aceleración del crecimiento de poblaciones de hormigas en el uso de Ss. El Bs presentó mayor similitud con el uso Fr, con un valor de 52,9\%.

El número de morfoespecies compartidas entre los diferentes usos de suelo fue mayor entre Fr y Bs, con 18 y entre Fr y Pc, con 15 morfoespecies compartidas. Armbrecht \& Chacón (1997) hallaron en bosques secos relictuales en el Valle del Cauca (Colombia), que los fragmentos boscosos mostraron mayor riqueza de especies y compartieron mayor número de especies con los hábitats aledaños. Los usos de suelo con menor número de morfoespecies compartidas fueron $\mathrm{Pb}$ y Ss, con 5 y entre $\mathrm{Pc}$ y Ss, con 7 morfoespecies compartidas y ésto se debe, en parte, a que el uso Ss fue el que menor riqueza de morfoespecies presentó.

Los géneros Amblyopone, Cyphomyrmex, Discothyrea, Gnamptogenys, Myrmicocrypta, Pachycondyla y Pyramica, se presentaron exclusivamente en un uso de suelo, bosque secundario (Tabla 1). Este resultado concuerda con los aspectos de la biología y microhábitat de estos siete géneros de hormigas, pues anidan en hojarasca, madera descompuesta, base de árboles, raíces expuestas o en tierra (Fernández, 2003), condiciones disponibles en sitios poco intervenidos, como es el caso del bosque secundario, en el cual, hay buena cantidad de hojarasca y material vegetal en descomposición.

Las morfoespecies presentes en los cinco usos de suelo fueron Hypoponera msp1, Pheidole msp4, Solenopsis msp1 y Solenopsis msp3 (Tabla 1). Para el caso del género Solenopsis presente en todos los usos del suelo, Silvestre et al. (2003) indicaron que forman grupos muy conspicuos en la hojarasca, construye nidos poco profundos y son indicadores de áreas con estrés ambiental. Con relación a la rareza, se observó que el total de morfoespecies presentes en un solo uso de suelo fueron 12 (Tabla 1), donde el 67\% se registró únicamente en bosque secundario (Bs). Cabe resaltar que estudios en otros bosques y cafetales de Colombia evidencian que los bosques preservan especies únicas y exclusivas más que los cafetales (Zabala et al. 2013); asimismo, a medida que un bosque se hace más rico tiene mayor cantidad de especies exclusivas de ese bosque, por lo cual, es reservorio de mayor diversidad y de especies raras (Armbrecht \& Chacón, 1997).

El género Pheidole presentó un mayor número de morfoespecies (22\%), seguido por los géneros Brachymyrmex, Solenopsis y Strumigenys, con el $8 \%$ cada uno, en tanto que el $49 \%$ de los géneros contienen 1 morfoespecie (Tabla 1). En el estudio realizado en la cuenca medio del río Calima, llevado a cabo por Aldana \& Chacón (1999), se registraron datos similares con relación al número de especies por género, donde el género Pheidole presentó un mayor número de especies. Vanegas \& Sepúlveda (2008), en la Estación Cotové, no registraron, para los usos de suelo $\mathrm{Fr}$ y Bs, un género predominante en el número de morfoespecies, teniendo el género Pheidole un $8,7 \%$, de las 23 morfoespecies registradas. Considerando la presencia de los géneros por uso de suelo (Tabla 1), los géneros Pheidole, Solenopsis y Hypoponera estuvieron presentes en los cinco usos de suelo. El género Pheidole, se registró más frecuentemente en frutales (Fr) y en bosque secundario (Bs); Solenopsis, se manifestó más en el uso Bs. De acuerdo con Williams (1994), los géneros Pheidole y Solenopsis forman parte de las especies con múltiples reinas y nidos que, frecuentemente, dominan los hábitats, gracias a su gran potencial de crecimiento y de extensión espacial.

Al comparar los géneros de hormigas registrados por Vanegas \& Sepúlveda (2008) en frutales con los géneros encontrados en el presente estudio en el mismo uso de suelo ( $\mathrm{Fr}$ ), en ambos estudios se registraron Crematogaster sp., Ectatomma sp., Mycocepurus sp., Pheidole sp. y Solenopsis sp. y otros géneros, se registraron por primera vez en estos sustratos, como: Wasmannia sp., Strumigenys sp., Rogeria sp., Pachycondyla sp., Paratrechina sp., Octostruma sp., Hypoponera sp. $y$ Anochetus sp. (Tabla 3). Respecto al bosque secundario, Vanegas \& Sepúlveda (2008) reportaron los géneros Octostruma sp., Pachycondyla sp., Pheidole sp. y Solenopsis sp. Por su parte, en el presente estudio, se hallaron 15 géneros diferentes y 10 de los géneros encontrados en 2008, no fueron ubicados en el presente trabajo para el uso de suelo de BS (Tabla 3). Estas variaciones pueden estar asociadas a las diferencias entre métodos de registro de las especies y al mantenimiento, a través del tiempo de estas coberturas vegetales, que favorecen el establecimiento de las especies en los territorios; una fracción considerable del ensamblaje de hormigas, se encuentra solo en etapas avanzadas de sucesión, lo que demuestra la importancia de los hábitats secundarios, para mantener la biodiversidad en los bosques secos (Marques et al. 2017).

Estructura trófica de la comunidad de hormigas: Los omnívoros representaron la mayor proporción de morfoespecies $(51,4 \%)$, seguido por depredadores $(40,5 \%)$ y cultivadores de hongos $(8,1 \%)$, de las morfoespecies registradas (Tabla 1). En el uso de bosque secundario (Bs), se registró mayor número de morfoespecies depredadoras que en otros usos (Figura 2a). Debido a la mayor frecuencia de asociaciones mutualistas entre plantas y hormigas, en las que las hormigas viven o se alimentan sobre las plantas y no se comportan como herbívoros en el sentido general, sino que ofrecen algún tipo de beneficio (Ibarra \& Dirzo, 1990).

Por su parte, pastos de corte (Pc) fue el uso de suelo que presentó más registros de morfoespecies del gremio omnívoros (Figura 2a). Ramírez \& Calle (2003) afirman que, en los pastizales, las hormigas cumplen funciones relevantes, como consumidores primarios y secundarios. Las morfoespecies cultivadoras de hongos, se presentaron en porcentajes menores al 10\% de los registros en los usos Fr, Ss y Bs y no se registró ninguna morfoespecie para los usos $\mathrm{Pb}$ y Pc (Figura 2a). 
Tabla 3. Presencia $(\mathrm{X})$ y ausencia de géneros de hormigas en la Estación Agraria Cotové en frutales ( $\mathrm{Fr}$ ) y bosque secundario (Bs) (presente estudio). *Sitios estudiados por Vanegas \& Sepúlveda (2008).

\begin{tabular}{|c|c|c|c|c|}
\hline Género & Fr & Lote A* & Bs & Lote B* \\
\hline Acromyrmex & & $\mathrm{X}$ & & $\mathrm{X}$ \\
\hline Acropyga & & & $\mathrm{X}$ & \\
\hline Amblyopone & & & $\mathrm{X}$ & \\
\hline Anochetus & $\mathrm{X}$ & & $\mathrm{X}$ & \\
\hline Apterostigma & & & & $\mathrm{x}$ \\
\hline Azteca & & $\mathrm{X}$ & & $\mathrm{X}$ \\
\hline Brachymyrmex & & & $\mathrm{X}$ & \\
\hline Camponotus & & $\mathrm{x}$ & & $\mathrm{x}$ \\
\hline Crematogaster & $\mathrm{X}$ & $\mathrm{X}$ & & $\mathrm{X}$ \\
\hline Cyphomyrmex & & & $\mathrm{x}$ & \\
\hline Discothyrea & & & $\mathrm{X}$ & \\
\hline Ectatomma & $\mathrm{X}$ & $\mathrm{X}$ & & $\mathrm{X}$ \\
\hline Gnamptogenys & & & $\mathrm{X}$ & \\
\hline Hypoponera & $\mathrm{X}$ & & $\mathrm{X}$ & \\
\hline Labidus & & & $\mathrm{X}$ & \\
\hline Lepthotorax & & $\mathrm{X}$ & & \\
\hline Myсосеригиs & $\mathrm{X}$ & $\mathrm{X}$ & & $\mathrm{X}$ \\
\hline Myrmicocrypta & & & $\mathrm{x}$ & \\
\hline Octostruma & $\mathrm{X}$ & & $\mathrm{X}$ & $\mathrm{x}$ \\
\hline Odontomachus & & $\mathrm{X}$ & & $\mathrm{X}$ \\
\hline Pachycondyla & $\mathrm{X}$ & & $\mathrm{X}$ & $\mathrm{X}$ \\
\hline Paratrechina & $\mathrm{X}$ & & $\mathrm{X}$ & \\
\hline Pheidole & $\mathrm{X}$ & $\mathrm{X}$ & $\mathrm{X}$ & $\mathrm{X}$ \\
\hline Pseudomyrmex & & $\mathrm{X}$ & & $\mathrm{X}$ \\
\hline Pyramica & & & $\mathrm{X}$ & \\
\hline Rogeria & $\mathrm{X}$ & & $\mathrm{X}$ & \\
\hline Sericomyrmex & & & & $\mathrm{X}$ \\
\hline Solenopsis & $\mathrm{X}$ & $\mathrm{x}$ & $\mathrm{X}$ & $\mathrm{x}$ \\
\hline Strumigenys & $\mathrm{X}$ & & $\mathrm{X}$ & \\
\hline Tapinoma & & & & $\mathrm{x}$ \\
\hline Trachymyrmex & & $\mathrm{X}$ & & \\
\hline Wasmannia & $\mathrm{X}$ & & $\mathrm{X}$ & \\
\hline Total & 13 & 12 & 19 & 15 \\
\hline
\end{tabular}

**Lote A: cultivo de mango; **Lote B: fragmento de bosque (Lote A y B corresponde a los sitios estudiados por Vanegas \& Sepúlveda (2008), los cuales, son equivalentes a Fr y Bs del presente estudio). 


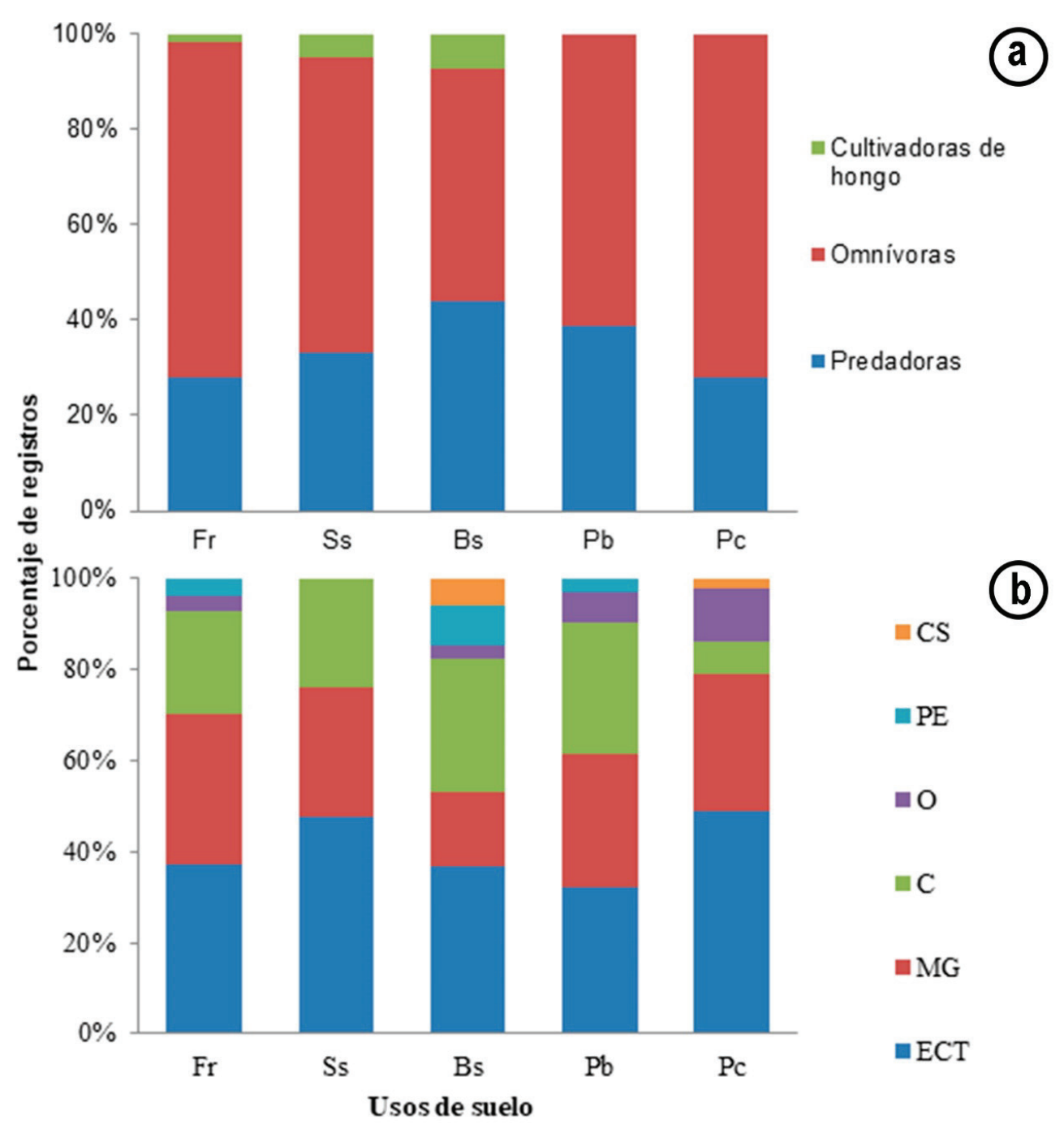

Figura 2. Porcentaje de registros de morfoespecies por: a. gremio trófico; b. grupo funcional, en los cinco usos de suelo de la Estación Agraria Cotové. Fr: Frutales; Ss: Sistema silvopastoril; Bs: Bosque secundario; Pb: Potreros de ganadería en baja densidad de árboles; Pc: Pastos de corte. CS: Camponotini subordinada; ECF: Especialistas de Clima frío; ECT: Especialistas de Clima Tropical; MG: Myrmicinae Generalista; O: Oportunistas; PE: Depredadores Especialistas.

Para todos los usos de suelo, se evidenció una mayor proporción de registros de morfoespecies del grupo funcional Especialistas de Clima Tropical (ECT) y se mostró diferencias significativas entre el uso $\mathrm{Pb}$, que presentó el menor número de registros de ECT, con los usos de Fr, Ss, Bs y Pc (Mann-Whitney, P=0,045, P=0,020, P=0,013, y $\mathrm{P}=0,005$, respectivamente) y entre el uso Pc y Bs (Mann-Whitney, $\mathrm{P}=0,045)$. Por su parte, el grupo MG fue el segundo en registros en los usos Fr, Ss y Pb (Figura 2b). El grupo funcional de morfoespecies cripticas $(\mathrm{C})$ presenta un porcentaje alto de registros en el uso Bs y $\mathrm{Pb}$, siendo, para este uso, el segundo en importancia, por encima del grupo MG y evidenciando diferencias significativas con el uso Pc (Mann-Whitney, $\mathrm{P}=0,044$, y $\mathrm{P}=0,019$, respectivamente), que presentó el menor valor de registros de morfoespecies crípticas. El grupo funcional Camponotini subordinada (CS) no fue registrado para los usos Fr, Ss y Pb; el grupo Oportunistas no se registró en Ss y el grupo Depredadores Especialistas (PE), no se consignó en los usos Ss y Pc (Figura 2b).

Los resultados de grupos funcionales, a nivel de morfoespecies, en el uso de Fr, en este estudio, son similares con los obtenidos por Vanegas \& Sepúlveda (2008), quienes encontraron, para este uso, mayor porcentaje del grupo de Especialistas de Clima Tropical (ECT), seguido del grupo MG; sin embargo, para el uso de Bs, hallaron predominio del grupo ECT, seguido de MG y oportunistas (O), mientras que el presente estudio presentó un predominio de ECT, seguido del grupo de especies crípticas. De acuerdo con Andersen (2000), los ECT son característicos de hábitats donde la abundancia de Dolichoderinae dominante es bajo y en cuanto a sus tolerancias 
de hábitat, ellos son, a menudo, hormigas no especializadas (excepto hormigas ejército y cultivadoras de hongos), mientras que las especies Crípticas son especies pequeñas, predominantemente myrmicines y ponerines, que nidifican y forrajean principalmente dentro del suelo, la capa vegetal y material en descomposición, siendo, en su mayoría, diversas y abundantes en hábitats del bosque y son un componente mayor de las hormigas de hojarasca, lo cual, puede explicar su mayor porcentaje en el uso de suelo de Bs.

A diferencia del estudio realizado por Vanegas \& Sepúlveda (2008), en la presente investigación no se encontraron los grupos Dolichoderinae dominante (DD), Especialistas de clima frío (ECF) y Especialistas de clima cálido (ECC). Para el caso del grupo funcional $\mathrm{DD}$, que integra géneros como Azteca, son altamente dominantes en el dosel de los árboles y que no se distribuyen homogéneamente, pero no fue hallado en este trabajo, puesto que el muestreo fue realizado en el suelo y no sobre los estratos arbóreos. Cabe mencionar que Vanegas \& Sepúlveda (2008) incluyeron al género Solenopsis, dentro del grupo ECC, pero según Andersen (2000), este género se agrupa mejor dentro del grupo funcional de ECT.

Con relación a las hormigas del grupo funcional Oportunista, hubo un mayor porcentaje de géneros en $\mathrm{Pc}$ y $\mathrm{Pb}$ y puede ser debido a que las hormigas oportunistas, por no presentar especialización en gremios tróficos o de nidificación, comúnmente, su distribución está influenciada por la competencia ejercida por las demás hormigas presentes en el ecosistema, predominando en lugares donde el disturbio limita la productividad y la diversidad de las hormigas (Vanegas \& Sepúlveda, 2008).

Se sugiere utilizar diferentes métodos de captura manual, trampas de caída y muestreo de suelo para un mejor reconocimiento de la mirmecofauna. Se hace prioritario proteger las áreas de vegetación nativa del uso de Bs y establecer nuevas áreas destinadas a usos de conservación, aumentando las áreas de vegetación nativa propia de bosques secos tropicales en las áreas de ladera que, actualmente, son usadas para el cultivo de frutales, puesto que en estos hábitats se observó la mayor riqueza de morfoespecies y que se establezcan adecuadas prácticas de manejo agropecuario, que permitan la conservación de la diversidad a nivel regional, incluyendo la diversidad biológica y los servicios ecosistémicos, que de ella se derivan, como una variable importante en la formulación de los planes de manejo y desarrollo de actividades productivas de la Estación.

Agradecimientos: A la Vicerrectoría de Investigación de la Universidad Nacional de Colombia. Conflicto de intereses: El manuscrito fue preparado y revisado con la participación de todos los autores, quienes declaran que no existe conflicto de intereses que ponga en riesgo la validez de los resultados presentados.

\section{REFERENCIAS}

1. ALDANA, R.; CHACÓN, P. 1999. Megadiversidad de hormigas (Hymenoptera: Formicidae) de la cuenca media del río Calima. Rev. Colomb. Entomol. 25(1-2):37-47.
2. ANDERSEN, A. 2000. A global ecology of rainforest ants: functional groups in relation to environmental stress and disturbance. En: Agosti, D.; Majer, J.; Alonso, E.; Schultz, T.R. (eds.). Ants: Standard methods for measuring and monitoring biodiversity. Biological Diversity Handbook Series. Smithsonian Institution Press. Washington D.C. p.25-34.

3. ARENAS-CLAVIJO, A.; ARMBRECHT, I. 2018. Gremios y diversidad de hormigas (Hymenoptera: Formicidae) en tres usos del suelo de un paisaje cafetero del Cauca-Colombia. Rev. Biol. Trop. 66(1):48-57.

4. ARMBRECHT, I.; CHACÓN, P. 1997. Composición y diversidad de hormigas en bosques secos delictuales y sus alrededores, en el Valle del Cauca, Colombia. Rev. Colomb. Entomol. 23(1-2):45-50.

5. BROWN, W. 2000. Diversity of ants. En: Agosti, D.; Majer, J.; Alonso, E.; Schultz, T.R. (eds). Ants: Standard methods for measuring and monitoring biodiversity. Biological Diversity Handbook Series. Smithsonian Institution Press. Washington D.C. p.45-79.

6. BUSTOS, J.; CHACÓN, P. 1997. Mirmecofauna y perturbación en un bosque de niebla Neotropical (Reserva Natural Hato Viejo, Valle del Cauca, Colombia). Rev. Biol. Trop. 45:259266.

7. FERNÁNDEZ, F. 2003. Claves y sinópsis de las subfamilias y géneros. En: Fernández, F. (ed). Introducción a las hormigas de la región Neotropical. Instituto de Investigación de Recursos Biológicos Alexander von Humboldt. Bogotá D.C., Colombia. p.261-331.

8. FOLEY, J.A.; RAMANKUTTY, N.; BRAUMAN, K.A.; CASSIDY, E.S.; GERBER, J.S.; JOHNSTON, M.; MUELLER, N.D.; O'CONNELL, C.; RAY, D.K.; WEST, P.C.; BALZER, C.; BENNETT, E.M.; CARPENTER, S.R.; HILL, J.; MONFREDA, C.; POLASKY, S.; ROCKSTRÖM, J.; SHEEHAN, J.; SIEBERT, S.; TILMAN, D.; ZAKS, D.P.M. 2011. Solutions for a cultivated planet. Nature. 478:337-342. https://doi.org/10.1038/nature10452

9. HAMMER, Ø.; HARPER, D.; RYAN, P. 2001. PAST: Paleontological statistics software package for education and data analysis. Palaeontología Electrónica [Computer program]. Disponible desde Internet en: http:// palaeoelectronica.org/2001_1/past/issue1_01.htm (con acceso 08/03/2008).

10. IBARRA, M.; DIRZO, R. 1990. Plantas mirmecófilas arbóreas de la Estación de Biología "Los Tuxtlas", Veracruz, México. Rev. Biol. Trop. 38(1):79-82.

11. JIMÉNEZ-HEREDIA, Y.; MARTÍNEZ-BRAVO, C.M.; MANCERA-RODRÍGUEZ, N.J. 2010. Características 
físicas y químicas del suelo en diferentes sistemas de uso y manejo en el Centro agropecuario Cotové, Santa Fe de Antioquia, Colombia. Rev. Suelos Ecuatoriales. 40:176-188.

12. KASPARI, M. 2003. Introducción a la ecología de hormigas. En: Fernández, F. (ed). Introducción a las hormigas de la región Neotropical. Instituto de Investigación de Recursos Biológicos Alexander von Humboldt. Bogotá D.C., Colombia. p.97-108.

13. MARQUES, T.; ESPIRÍTO-SANTO, M.M.; NEVES, F.S.; SCHOEREDER, J.H. 2017. Ant assemblage structure in a secondary tropical dry forest: the role of ecological succession and seasonality. Sociobiology. 64(3): 261-275. https://doi.org/10.13102/sociobiology.v64i3.1276

14. MARTÍNEZ-BRAVO, C.M.; MANCERA-RODRÍGUEZ, N.J.; BUITRAGO-FRANCO, G. 2013. Diversidad de aves en el Centro Agropecuario Cotové, Santa Fe de Antioquia, Colombia. Rev. Biol. Trop. 61(4):1597-1617. https://doi. org $/ 10.15517 /$ rbt.v61i4.12801

15. PALACIO E. 1997. Hormigas de Colombia IV. Dos nuevas especies de Octostruma (Hymenoptera: Formicidae: Basicerotini). Caldasia. 19(3):409-418.

16. PALACIO, F.; FERNÁNDEZ, F. 2003. Claves para las subfamilias y géneros. En: Fernández, F. (ed.). 2003. Introducción a las hormigas de la región Neotropical. Instituto de Investigación de Recursos Biológicos Alexander von Humboldt. Bogotá D.C., Colombia. p.233-260.

17. PARDO, L.; VÉLEZ, C.; SEVILLA, F.; MADRID, O. 2006. Abundancia y biomasa de macroinvertebrados edáficos en la temporada lluviosa, en tres usos de la tierra, en los Andes colombianos. Acta Agronómica. 55(1):43-54.

18. PENNINGTON, T.; PRADO, D.E.; PENDRY, C.A. 2000. Neotropical seasonally dry forests and Quaternary vegetation changes. J. Biogeography. 27(2):261-273.

19. RAMÍREZ, M.; CALLE, Z. 2003. Ecología de hormigas en sistemas silvopastoriles. En: Sánchez, M.D.; RosalesMéndez, M. (eds). Agroforestería para la producción animal en América Latina - II. Dirección de Producción y Sanidad Animal FAO. Roma, Italia. p.65-74.

20. SERNA, F; VERGARA, E. 2007a. Historia natural de las hormigas cazadoras del departamento de Antioquia (Colombia). En: Jiménez, E., Fernández, F.; Arias, T.M.; Lozano-Zambrano, F.H. (eds). Sistemática, biogeografía y conservación de las hormigas cazadoras de Colombia. Instituto de Investigación de Recursos Biológicos Alexander von Humboldt. Bogotá D.C., Colombia. p.573-591.

21. SERNA, F.; VERGARA, E. 2007b. Hormigas cazadoras de Porce (Antioquia, Colombia). En: Jiménez, E., Fernández, F; Arias, T.M.; Lozano-Zambrano, F.H. (eds). Sistemática, biogeografía y conservación de las hormigas cazadoras de Colombia. Instituto de Investigación de Recursos Biológicos Alexander von Humboldt. Bogotá D. C., Colombia. p.553-572.

22. SERNA, F.; VERGARA, E. 2001. Claves para la Identificación de Subfamilias y Géneros de Hormigas de Antioquia y Chocó, Colombia. Revista del I.C.N.E. 7(1):5-41.

23. SILVESTRE, R.; BRANDÃO, C.R.F.; ROSA DA SILVA, R. 2003. Grupos funcionales de hormigas: el caso de los gremios del Cerrado. En: Fernández, F. (ed). Introducción a las hormigas de la región Neotropical. Instituto de Investigación de Recursos Biológicos Alexander von Humboldt. Bogotá D.C., Colombia. p.113-146.

24. TORO, E.; ORTEGA, O.E. 2006. Composición y diversidad de hormigas (Hymenoptera: Formicidae) en algunas áreas protegidas del Valle de Aburrá. Rev. Colomb. Entomol. 32(2):214-220.

25. VANEGAS, M.; SEPÚlVEDA, P. 2008. Comparación de la riqueza y diversidad de hormigas en dos tipos de hábitat en un bosque seco tropical. Boletín Informativo Sociedad Colombiana de Entomología. 2:2-9.

26. VERGARA, N.E.V.; ECHAVARRÍA, S.H.; SERNA, C.F.J. 2007. Hormigas (Hymenoptera Formicidae) asociadas al arboretum de la Universidad Nacional de Colombia, núcleo el volador, Medellín. Boletín de la Sociedad Entomológica Aragonesa. 40:497-505.

27. WILLIAMS, D.F. 1994. Exotic ants: Biology, impact. and control of introduced species. Westview Press, Boulder, U.S.A. 322p.

28. ZABALA, G.A.; ARANGO, L.M.; CHACÓN, P. 2013. Diversidad de hormigas (Hymenoptera: Formicidae) en un paisaje cafetero de Risaralda, Colombia. Rev. Colomb. Entomol. 39(1):141-149.

29. ZAR, J.H. 1999. Biostatistical analysis. Prentice-Hall. New Jersey, U.S.A. 960p. 